\title{
IncRNA TUG1 promotes the brown remodeling of white adipose tissue by regulating miR-204-targeted SIRT1 in diabetic mice
}

\author{
YING ZHANG ${ }^{1,2}$, YUHANG MA ${ }^{2}$, MINGYU GU ${ }^{1,2}$ and YONGDE PENG ${ }^{1,2}$ \\ ${ }^{1}$ Department of Endocrinology and Metabolism, Shanghai General Hospital of Nanjing Medical University; \\ ${ }^{2}$ Department of Endocrinology and Metabolism, Shanghai General Hospital, Shanghai Jiaotong \\ University School of Medicine, Shanghai 200080, P.R. China
}

Received February 26, 2020; Accepted August 5, 2020

DOI: $10.3892 /$ ijmm.2020.4741

\begin{abstract}
The present study aimed to explore the mechanisms of the long non-coding RNA TUG/miR-204/SIRT1 axis in the pathogenesis of obesity. For this purpose, a diabetic mouse model was constructed using a high-fat diet and streptozocin, and the mice were treated with TUG1 virus via tail intravenous injection. Body weight, serum glucose levels, insulin levels and testicular fat, as well as the expression levels of TUG1, miR-204, Sirtuin 1 (SIRT1) and the AMP-activated protein kinase (AMPK)/acetyl-CoA carboxylase (ACC) signaling pathway were detected. Furthermore, the regulatory mechanisms of TUG1/SIRT1 and miR-204 in the development of diabetes were also explored. The results revealed that the overexpression of TUG1 significantly attenuated body weight, serum glucose levels, insulin tolerance and fatty accumulation in diabetic mice. Furthermore, the overexpression of TUG1 significantly increased the expression SIRT1, adipose triglyceride lipase (ATGL), peroxisome proliferator-activated receptor $\alpha(\mathrm{PPAR} \alpha)$, peroxisome proliferator-activated receptor gamma coactivator 1- $\alpha$ (PGC-1 $\alpha$ ) and uncoupling protein-1 (UCP-1), as well as the phosphorylation levels of AMPK and ACC, and decreased the expression of miR-204 in adipose tissues and 3T3-L1 cells. miR-204 inhibitor increased the expression SIRT1, ATGL, PPAR $\alpha$, PGC-1 $\alpha$ and UCP-1, as well as the phosphorylation levels of AMPK and ACC, and decreased the expression of miR-204 in the 3T3-L1 cells; however, the silencing of SIRT1 attenuated these effects. On the whole, the findings of the present study demonstrate that IncRNA TUG1 significantly reverses the development of diabetes by
\end{abstract}

Correspondence to: Dr Yongde Peng, Department of Endocrinology and Metabolism, Shanghai General Hospital, Shanghai Jiaotong University School of Medicine, 100 Haining Road, Shanghai 200080, P.R. China

E-mail: peng017@outlook.com

Key words: type-2 diabetes mellitus, adipose, Sirtuin 1, AMP-activated protein kinase/acetyl-CoA carboxylase pathway, long non-coding RNA TUG1, miR-204 downregulating the expression of miR-204, and upregulating its targeted SIRT1/AMPK/ACC signaling pathway.

\section{Introduction}

Diabetes is the leading cause of morbidity and mortality worldwide, and has affected $>415$ million of the world population (1). Type-2 diabetes mellitus (T2DM) is characterized by an impaired insulin secretion and insulin resistance in adipose, liver and muscle tissues (2). Obesity is the principal causative factor for the development of multiple metabolic diseases, including cardiovascular disease, neuropathy, retinopathy, chronic kidney disease and nephropathy, as well as the increasing healthcare costs (3). Various genetic and environmental factors may contribute to the progression of T2DM; however, the mechanisms responsible for the development of T2DM remain unclear (4).

It has previously been demonstrated that obesity is one of the most crucial factors that contributes to T2DM (5). As an energy depository, adipose tissue also produces several bioactive molecules/adipokines to regulate insulin sensitivity, glucose metabolism and lipid metabolism-modulating processes, such as adipogenesis, angiogenesis, hypertension, bone morphogenesis and cell adhesion (6). The activation of brown adipose tissue (BAT) can ameliorate metabolic abnormalities in obesity and can thus resolve the common complications associated with obesity, including T2DM (7). The study conducted by Zhao et al revealed that the deletion of $\alpha / \beta$-hydrolase domain 6 promoted the browning of the adipose tissue and prevented T2DM (8). Insulin suppresses lipolysis in white adipose tissue (WAT) to reduce fatty acid flux to the liver, resulting in obesity and T2DM (9). In spite of these findings however, the exact mechanisms underlying the association between obesity and diabetes remain largely unknown.

Long non-coding RNAs (lncRNAs) are a class of protein incapability transcripts of 200 nucleotides in length, and have been identified as important regulators of diabetes (10). Carter et al reported that circulating lncRNA GAS5 expression was associated with the population of T2DM (11). The inhibition of IncRNA NONRATT021972 alleviates diabetic neuropathic pain via the $\mathrm{P} 2 \mathrm{X} 3$ receptor in the dorsal root ganglia (12). Leti and DiStefano reported that lncRNAs function as diagnostic and therapeutic targets in T2DM and related 
complications (13). Recently, the TUG1/TraF5 signaling pathway has been reported to be involved in podocyte apoptosis in rats with diabetic nephropathy through the modulation of extracellular matrix accumulation $(14,15)$. However, the function of TUG1 in diabetes has rarely been reported, at least to the best of our knowledge.

Sirtuin 1 (SIRT1) is a member of the mammalian sirtuins, and is characterized by $\mathrm{NAD}^{+}$-dependent deacetylases and ADP-ribosyl transferases that function in multiple cellular process, including DNA repair, gene silencing and metabolic regulation (16). SIRT1 has been reported to be a therapeutic target for T2DM (17), and has the function of improving energy efficiency and preventing diabetes in mice (18). miR-204 has been reported to play key roles in the processes of obesity and diabetes (19). However, the mechanisms of the miR-204-SIRT1 axis remain unclear as regards the regulation of diabetes.

In the present study, a diabetic mouse model was constructed to explore the function of the lncRNA TUG1, SIRT1 and miR-204 in diabetes. In addition, the regulatory mechanisms of and associations between TUG1, SIRT1 and miR-204 were confirmed in 3T3-L1 cells, followed by pathway investigation. According to these investigations, it is hoped the findings of the present study may provide novel new insight into the pathogenesis and treatment of diabetes.

\section{Materials and methods}

Construction of the diabetic mouse model. The animal protocols used in the present study were authorized by the Ethics Committee of the Shanghai General Hospital of Nanjing Medical University. The use of animals was also approved by the same Ethics Committee. A total of 100 C57BL/6 J mice (5 weeks, weighing $24 \pm 2 \mathrm{~g}$ ), were purchased from Slac Laboratory Animal Co., Ltd., and housed under a 12-h light/dark cycle, SPF conditions, with free access to food and water. Following 1 week of adaptation, these mice were randomly divided into 2 groups. Specifically, mice in the control group $(\mathrm{n}=20)$ were fed a normal diet $(3 \% \mathrm{kcal}$ from fat, Harlan Teklad), and mice in the model group $(n=80)$ were fed a high-fat diet (45\% kcal from fat, D12451, Research Diet Inc.). After 6 weeks, the mice in the model group were intraperitoneally injected with streptozotocin $(50 \mathrm{mg} / \mathrm{kg}$; EMD Chemicals, Inc.; Merck KGaA) to establish the diabetic model. Blood samples (0.1-0.2 ml for each mouse) were then collected via tail vein blood collection and blood glucose levels were measured weekly. When the blood glucose of mice was $>11.0 \mathrm{mmol} / \mathrm{l}$, the insulin level was considered to be markedly increased. The mice in the model group were then further randomly divided into 3 groups as follows: The model $(n=23)$, TUG1 NC $(n=23)$ and TUG1 $(\mathrm{n}=24)$ groups. The mice in the TUG1 NC group were injected with the TUG1 NC virus via the tail vein, while the mice in the TUG1 group were injected with the TUG1 overexpression virus via the tail vein. The lentivirus of TUG1 or TUG1 NC were purchased from Promega (Beijing Biotech Co., Ltd.). After 8 weeks, the mice were anesthetized by an intraperitoneal injection with $10 \%$ chloral hydrate $(300 \mathrm{mg} / \mathrm{kg}$; no peritonitis or any signs of discomfort were observed in the mice) and sacrificed by cervical dislocation, blood samples (1.0-1.2 $\mathrm{ml}$ per mouse) were collected via the tail vein prior to euthanasia by cervical dislocation. Fatty tissues were also collected for the following investigations. During the experimental process, mouse behavior was routinely monitored, the weight of the mice was measured each day and blood glucose levels were measured weekly. A total of 8 mice ( 3 in the model group, 2 in the TUG1 NC group and 3 in the TUG1 group) were excluded due to severe infection. For the excluded mice, they were anesthetized by an intraperitoneal injection with $10 \%$ chloral hydrate $(300 \mathrm{mg} / \mathrm{kg})$ and sacrificed by cervical dislocation.

Detection of associated indices in mice with obesity. In the process of the establishment of the model, body weight and serum glucose levels were detected each week, and the fasting blood glucose (FBG), triglyceride (TG), glycerol and non-esterified fatty acid (NEFA) levels were determined at the 8th week using ELISA kits (Sigma-Aldrich; Merck KGaA), according to the manufacturer's instructions.

Immunohistochemistry (IHC). Following collection, part of the tissues was fixed with $4 \%$ paraformaldehyde (PFA) at $4^{\circ} \mathrm{C}$ overnight. The tissues were then dehydrated by gradient ethanol, permeabilized by xylene and embedded in paraffin. Subsequently, these tissues were cut into $4-\mu \mathrm{m}$-thick sections. The paraffin tissue slices were then dewaxed by heating, and rehydrated with gradient ethanol. EDTA antigen reduction solution (Sigma-Aldrich; Merck KGaA) was then used to repair the antigens on the sections in a microwave oven at $40^{\circ} \mathrm{C}$, followed by natural cooling. After washing twice with phosphate-buffered saline (PBS, 3 min per time), the sections were blocked with $10 \%$ bovine serum albumin for $30 \mathrm{~min}$, and incubated with uncoupling protein-1 (UCP-1) antibody (1:200; cat. no. ab10983, Abcam) at $4^{\circ} \mathrm{C}$ overnight. After washing 3 times with PBS ( 3 min per time), the sections were incubated with the secondary antibody (1:1,000; cat. no. ab205718, Abcam) at room temperature for $50 \mathrm{~min}$, followed by hematoxylin and eosin (H\&E) staining at room temperature for $10 \mathrm{~min}$. Finally, the sections were washed 3 times with PBS ( 5 min per time), mounted with neutral resins and analyzed under a light microscope (Nikon Eclipse 1000, Nikon Corporation).

Cell culture and transfection. The 3T3-L1 cells were purchased from the Chinese Center of Type Culture Collection and maintained in Dulbecco's modified Eagle's medium (DMEM; Gibco; Thermo Fisher Scientific, Inc.), supplemented with 10\% fetal bovine serum (Gibco; Thermo Fisher Scientific, Inc.), $100 \mu \mathrm{g} / \mathrm{ml}$ of streptomycin (Sigma-Aldrich; Merck $\mathrm{KGaA}$ ) and $100 \mu \mathrm{g} / \mathrm{ml}$ of penicillin (Sigma-Aldrich; Merck $\mathrm{KGaA}$ ), in a humidity incubator with $5 \% \mathrm{CO}_{2}$ at $37^{\circ} \mathrm{C}$. When the cells grew to $70 \%$ confluency, they were transfected with miR-204 mimics, miR-204 inhibitor, mimics/inhibitor NC, si-SIRT1 and si-SIRT1 NC, as well as the pshRNA-H1-Luc lentivirus vector (all $10 \mathrm{nM}$ for the above) using Lipofectamine 2000 (Invitrogen; Thermo Fisher Scientific, Inc.), according to manufacturer's protocol. For transfection, the mimics/inhibitor, siRNA or vectors were incubated with Lipofectamine 2000 solution at room temperature for $20 \mathrm{~min}$, following by $48 \mathrm{~h}$ of incubation in a humidity incubator with $5 \% \mathrm{CO}_{2}$ at $37^{\circ} \mathrm{C}$. The miR-204 mimic, miR-204 inhibitor, NCs and plasmids were purchased from KeyGen Biotech. These cells were used for the downstream investigation at $48 \mathrm{~h}$ following transfection. The 
Table I. List of primers used for RT-qPCR.

\begin{tabular}{lll}
\hline Gene & \multicolumn{1}{c}{ Forward primer } & \multicolumn{1}{c}{ Reverse primer } \\
\hline TUG1 & 5'-CTGAAGAAAGGCAATCCATC-3' & 5'-GTAGGCTACTACAGGTCATTTG-3' \\
SIRT1 & 5'-TGCTGGCCTAATAGAGTGGCA-3' & 5'-CTCAGCGCCATGGAAAATGT-3' \\
miR-204 & 5'-ACACTCCAGCTGGGTTCCCTTTTCATCCT-3' & 5'-CTCAACTGGTGTCGTGGAGTCGG-3' \\
ATGL & 5'-CCCACTTCAACTCCAAGGACGA-3' & 5'-GCAGGTTGTCTGAAATGCCACC-3' \\
PPAR $\alpha$ & 5'-TCGGCGAACTATTCGGCTG-3' & 5'-GCACTTGTGAAAACGGCAGT-3' \\
PGC- $\alpha$ & 5'-AAACTTGCTAGCGGTCCTCA-3' & 5'-TGGCTGGTGCCAGTAAGAG-3' \\
UCP-1 & 5'-CAAAAACAGAAGGATTGCCGAAA-3' & 5'-TCTTGGACTGAGTCGTAGAGG-3' \\
U6 & 5'-CTCGCTTCGGCAGCACA-3' & 5'-AACGCTTCACGAATTTGCGT-3' \\
GAPDH & 5'-ATCACTGCCACCCAGAAGAC-3' & 5'-TTTCTAGACGGCAGGTCAGG-3' \\
\hline
\end{tabular}

miR-204 inhibitor and inhibitor NC were provided by KeyGen Biotech with no sequence information. The sequences for miR-204 NC, miR-204 mimics, si-SIRT1 NC and si-SIRT1are listed as follows: miR-204 mimics NC, 5'-AUCCGGAAU UUUAAGCCUAUCUGA-3'; miR-204 mimics, 5'-UUCCCU UUGUCAUCCUAUGCCU-3'; si-SIRT1 NC, 5'-UUAUGC UAGCAUCGAUCAAGC-3'; si-SIRT1, 5'-GCAGGTTGC AGGAATCCAAAG-3'. To establish the high glucose-induced 3T3-L1 fatty model, 3T3-L1 cells were exposed to $25 \mathrm{mM}$ glucose [D(+) glucose, G-5400, Sigma-Aldrich; Merck KGaA] at $48 \mathrm{~h}$ post-transfection for an additional 48 hours. The control group was left untreated.

Dual-luciferase reporter activity assay. Briefly, the 3T3-L1 cells were seeded in 48-well plates, and maintained in $200 \mu \mathrm{l}$ of DMED containing $10 \%$ FBS overnight. For the determination of the regulatory mechanisms between miR-204 and TUG1, the cells were transfected using Lipofectamine 2000 (Invitrogen; Thermo Fisher Scientific, Inc.), according to manufacturer's protocol, and divided into the following groups: The pGL6-TUG1-WT+miR-204 NC, pGL6-TUG1-WT + miR-204 mimic, pGL6-TUG1-MUT + miR-204 NC and pGL6-TUG1-MUT + miR-204 mimic groups. For the regulatory confirmation between mR-204 and SIRT1, the cells were respectively transfected according to the requirement of each assay, and divided into the following groups: The pGL6-SIRT1-WT + miR-204 NC,pGL6-SIRT1-WT + miR-204 mimic, pGL6-SIRT1-MUT + miR-204 NC and the pGL6-SIRT1-MUT + miR-204 mimic groups. The plasmids and miR-204 mimics were purchased from Promega (Beijing Biotech Co., Ltd.). At $48 \mathrm{~h}$ following transfection, these cells were washed 3 times with PBS, and the luciferase activity was measured using the Dual-Luciferase reporter assay system (GloMax; Promega Corporation), according to manufacturer's protocol. The Firefly luciferase activity was normalized to the Renilla luciferase activity.

Reverse transcription-quantitative polymerase chain reaction (RT-qPCR). Total RNA was isolated from the tissues or cell samples using TRIzol reagent (Takara Biotechnology Co., Ltd.), according to manufacturer's protocols. Subsequently, $2 \mu \mathrm{g}$ of total RNA were reverse transcribed into first-strand cDNA using the 5X PrimeScript RT Master Mix (Takara
Biotechnology Co., Ltd.) or the TaqMan MicroRNA Reverse Transcription kit (Applied Biosystems), according to manufacturer's instructions. The amplifications of the genes or miRNAs were performed using SYBR-Green (Varioskan Flash; Thermo Fisher Scientific, Inc.) under the following conditions: $95^{\circ} \mathrm{C}$ for $10 \mathrm{~min}, 95^{\circ} \mathrm{C}$ for $3 \mathrm{~min}$, and 40 cycles of $95^{\circ} \mathrm{C}$ for $10 \mathrm{sec}$ and $60^{\circ} \mathrm{C}$ for $30 \mathrm{sec}$. The housekeeping gene, GAPDH, was set as the gene internal control, and U6 was used as the miRNA internal control. The primers used for RT-qPCR are listed in Table I.

Western blot analysis. Protein was extracted from the tissue and cell samples using RIPA lysis buffer containing a protease inhibitor cocktail (Roche Diagnostics), and qualified using the BCA kit (Pierce; Thermo Fisher Scientific, Inc.). Proteins (50 $\mu \mathrm{g}$ per lane) were separated by $10 \%$ sodium dodecyl sulfate-polyacrylamide gel electrophoresis (SDS-PAGE), and transferred onto polyvinylidene difluoride (PVDF) membranes (Amersham Biosciences). The membranes were then blocked with TBS Tween containing 5\% non-fat milk (Inner Mongolia Yili Industrial Group Co., Ltd.) at room temperature for $1 \mathrm{~h}$, followed by incubation with primary antibodies to SIRT1 (1:800, cat. no. ab110304, Abcam), adipose triglyceride lipase (1:1,000, ATGL; cat. no. ab207799, Abcam), MAPK (1:1,000, cat. no. ab31828, Abcam), p-acetyl-CoA carboxylase (1:800, ACC; cat. no. ab68191, Abcam), ACC (1:800, cat. no. ab45174, Abcam), peroxisome proliferator-activated receptor $\alpha(1: 1,000$, PPAR $\alpha$; cat. no. ab215270, Abcam), peroxisome proliferator-activatedreceptorgammacoactivator $1-\alpha(1: 1,000, \mathrm{PGC}-1 \alpha$; cat. no. ab54481, Abcam), UCP-1 (1:1,000, cat. no. ab10983, Abcam) and GAPDH (1:2,000, cat. no. sc-365062, Santa Cruz Biotechnology, Inc.) at $4{ }^{\circ} \mathrm{C}$ overnight. The membranes were then incubated with goat anti-rabbit $\mathrm{IgG}$ and goat anti-mouse $\operatorname{IgG}(1: 2,000$, cat. no. ab205718 and cat. no. ab6708, Abcam) at room temperature for $1 \mathrm{~h}$, and developed using the ECL plus kit (PerkinElmer, Inc.). Densitometry was performed using the Alphalmager ${ }^{\mathrm{TM}} 2000$ Imaging System (Alpha Innotech Corporation).

Statistical analysis. All experiments in the present study were performed in triplicate, and data are presented as the means \pm standard deviation (SD). Statistical analyses in the present study were performed using GraphPad Prism 6.0. 
A

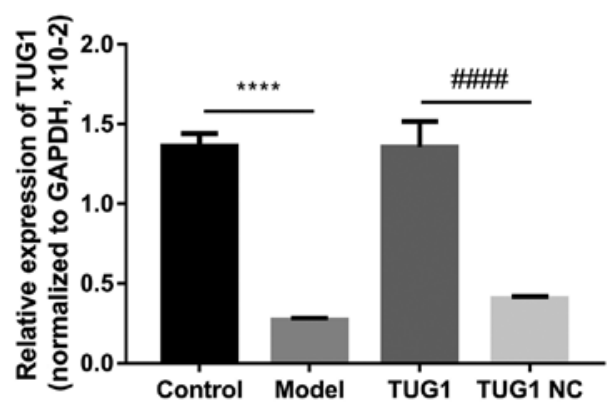

C

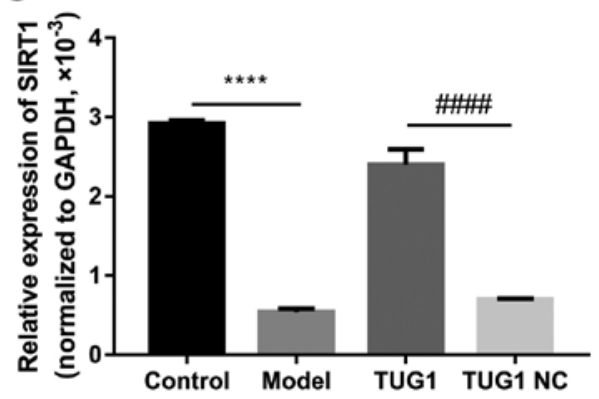

B

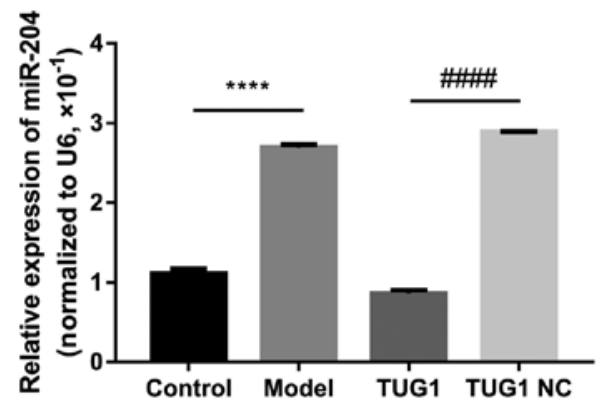

D

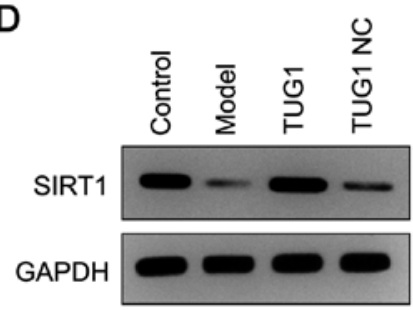

Figure 1. TUG1 decreases miR-204 expression, but promotes SIRT expression in diabetic mice. Relative expression of (A) TUG1, (B) miR-204 and (C) SIRT1 was evaluated by RT-qPCR. (D) The protein expression of SIRT1 was measured by western blot analysis. NC, negative control. Each RT-qPCR assay was

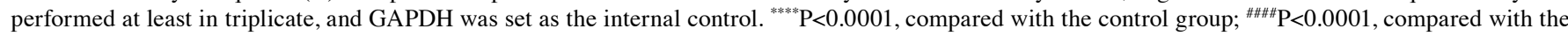
TUG1 NC group.

A

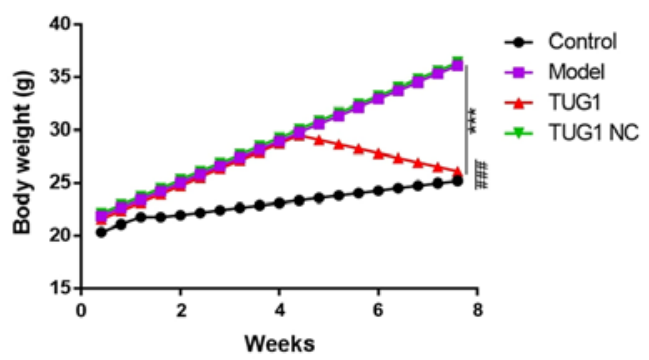

C

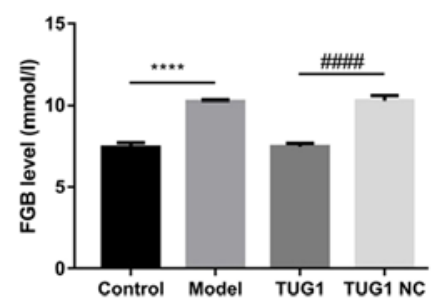

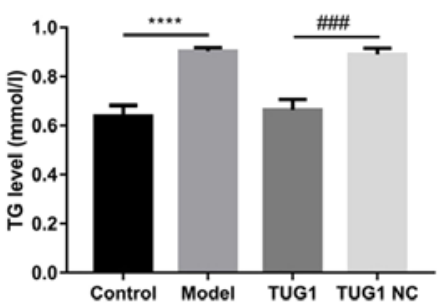

B

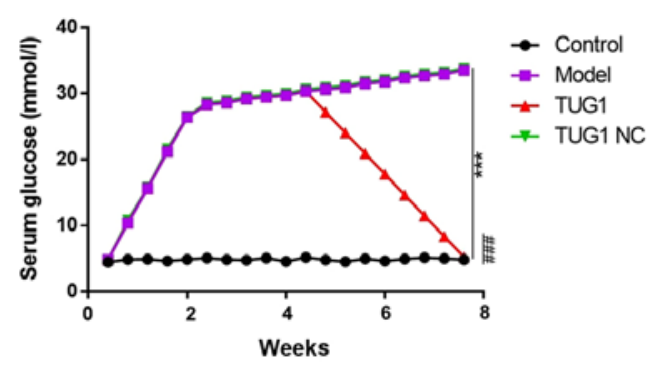

Figure 2. TUG1 improves weight accumulation and promotes adipose metabolism in diabetic mice. (A) Body weight of the diabetic mice in the control, model, TUG1 and TUG1 NC groups, respectively. (B) Serum glucose levels in these 4 groups. (C) Levels of serum fasting blood glucose (FBG), TG, glycerol and NEFA were detected. NC, negative control; TG, triglyceride; NEFA, non-esterified fatty acid. ${ }^{* * *} \mathrm{P}<0.001$ and ${ }^{* * * *} \mathrm{P}<0.0001$, compared with the control group;

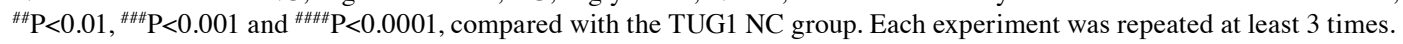

Statistical significance was assessed by a Student's t-test or one-way analysis of variance (ANOVA) with Turkey's post hoc tests, and $\mathrm{P}<0.05$ was considered to indicate a statistically significant difference.

\section{Results}

Overexpression of TUG1 decreases miR-204 expression, but increases SURT1 expression. After establishing the diabetic mouse model, the abdominal subcutaneous adipose tissues of the mice were obtained to detect the expression of miR-204 and SIRT1. The results of RT-qPCR revealed that TUG1 was significantly downregulated in the diabetic mice; however, the injection of TUG1 significantly increased the expression of TUG1 in the adipose tissues (all, $\mathrm{P}<0.05$; Fig. 1A). Furthermore, the expression of miR-204 was significantly increased in the adipose tissues of the diabetic mice, and the overexpression of TUG1 significantly inhibited the expression 
A
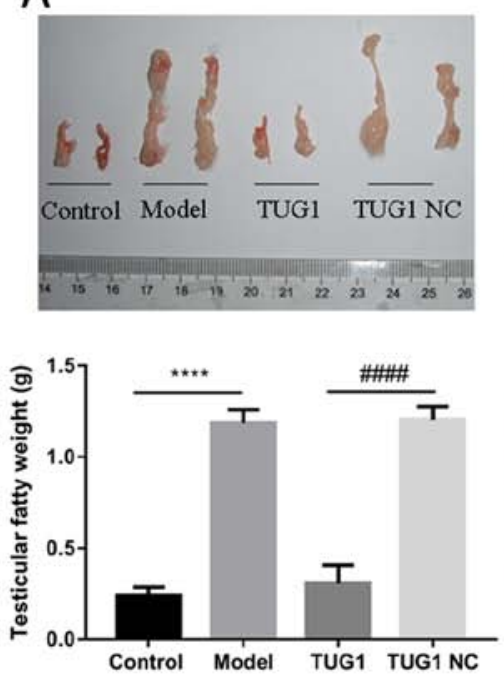

D
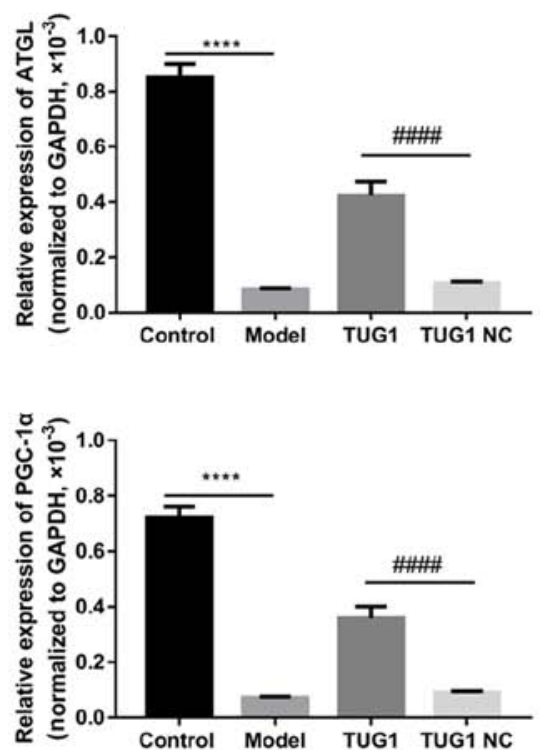

B

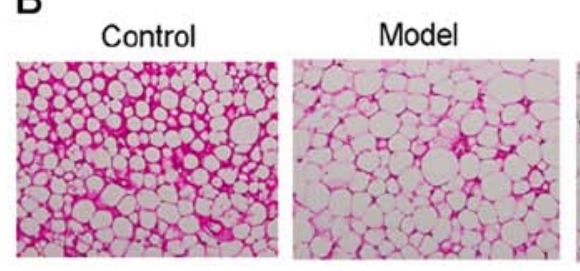

TUG1

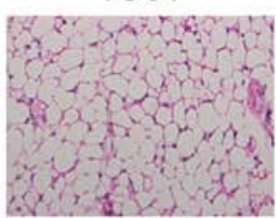

TUG1 NC

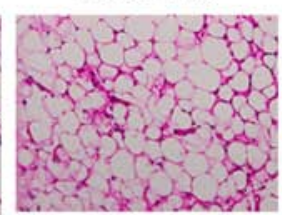

C
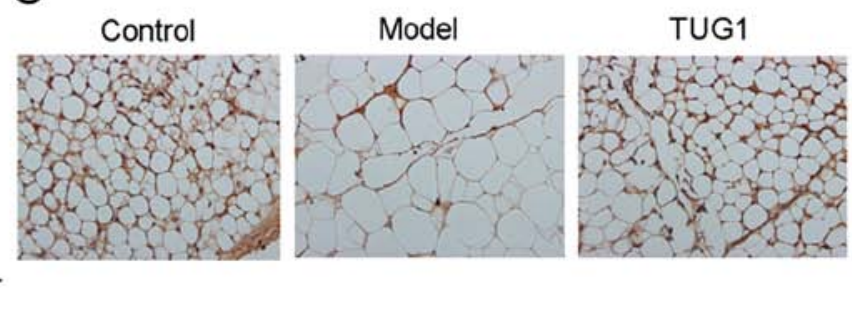

TUG1 NC

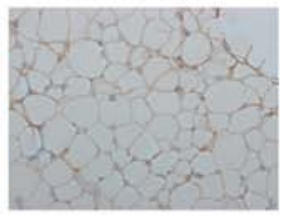

E
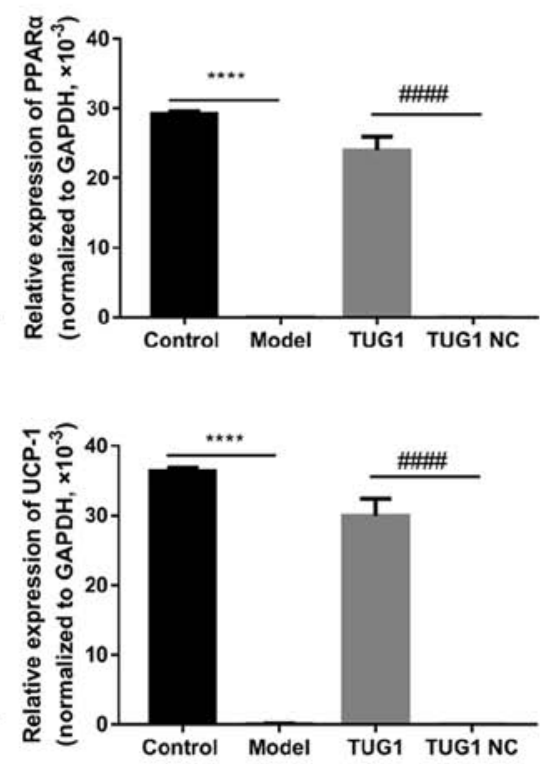

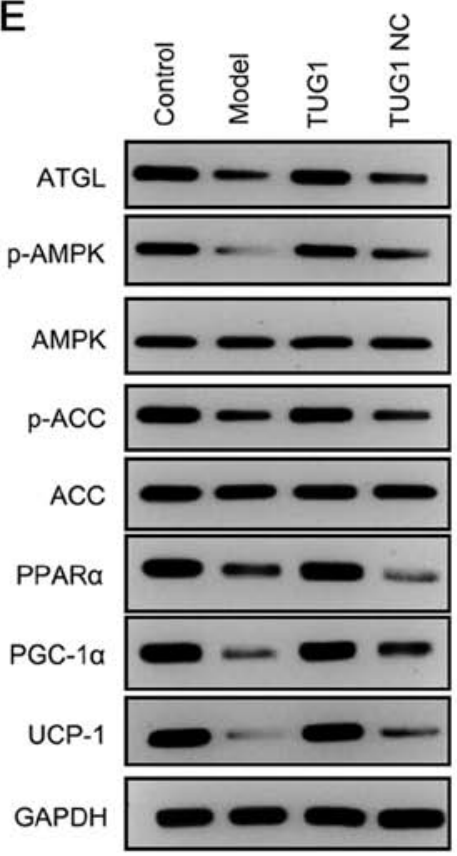

Figure 3. The overexpression of TUG1 reduces testicular adipose accumulation and promotes fat degradation, fatty acid oxidation and brown fat construction. (A) The weight of testicular fatty tissues was measured using ruler, and statistically analyzed in columns. (B) Hematoxylin and eosin (H\&E) staining was applied to examine the testicular fatty tissues. (C) The expression of UCP-1 in testicular fatty tissues was analyzed by immunohistochemistry (IHC). (D) The expression of ATGL, PGC-1 $\alpha$, PPAR $\alpha$ and UCP-1 was statistically evaluated by RT-qPCR. (E) The protein expression levels of AMPK, p-AMPK, ACC, p-ACC, ATGL, PGC-1 $\alpha$, PPAR $\alpha$ and UCP-1 were detected by western blot analysis. ATGL, adipose triglyceride lipase; PGC-1 $\alpha$, peroxisome proliferator-activated receptor gamma coactivator 1- $\alpha$; PPAR $\alpha$, peroxisome proliferator-activated receptor $\alpha$; UCP-1, uncoupling protein-1; AMPK, AMP-activated protein kinase; ACC, acetyl-CoA carboxylase; NC, negative control. ${ }^{* * * *} \mathrm{P}<0.0001$, compared with the control group; ${ }^{\# \# \# \#} \mathrm{P}<0.0001$, compared with the TUG1 NC group. Each assay, apart from western blot analysis, was performed in triplicate.

of miR-204 in the diabetic mice (all, $\mathrm{P}<0.05$; Fig. 1B). In addition, the mRNA and protein levels of SIRT1 were significantly downregulated in the diabetic mice; however, the upregulation of TUG1 markedly increased the expression of SIRT1 in the diabetic mice (all, $\mathrm{P}<0.05$; Fig. $1 \mathrm{C}$ and $\mathrm{D}$ ).

Overexpression of TUG1 attenuates the weight gain and abnormal metabolism of diabetic mice. As important indices in diabetes, the variations of weight and metabolism were also measured in the present mouse model. The results revealed that the injection of TUG1 significantly attenuated the elevated weight and serum glucose levels in the diabetic mice (all,
$\mathrm{P}<0.05$; Fig. $2 \mathrm{~A}$ and B). In addition, the TUG1 injection also markedly suppressed the upregulation of the diabetes-associated metabolic indices, including FBG, TG, glycerol and NEFA (all, P<0.05; Fig. 2C).

Overexpression of TUG1 promotes fat degradation, fatty acid oxidation and brown fat construction. In order to further reveal the biofunction of TUG1, the testicular adipose tissues of mice were collected, and it was found that the injection of TUG1 markedly attenuated the accumulation of testicular adipose tissue in diabetic mice (all, $\mathrm{P}<0.05$; Fig. $3 \mathrm{~A}$ ). H\&E staining revealed that the adipocytes were significantly larger 
A

hsa-miR-204-5p 3' UCCGUAUCCUACUG-UUUCCCUU 5'

TUG1 (1239-1260) 5' CACCATCGGGTGCCTGAACCGTG 3
B

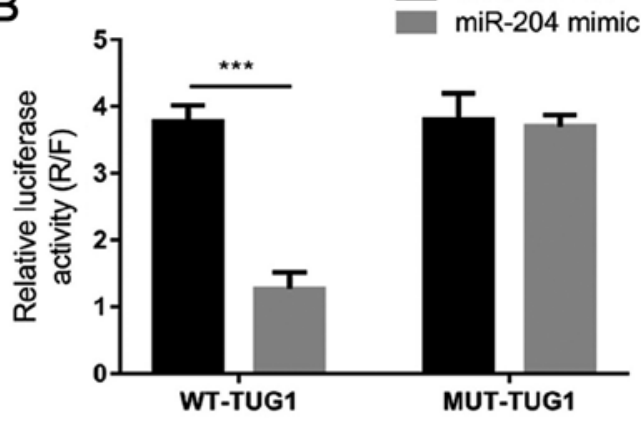

C

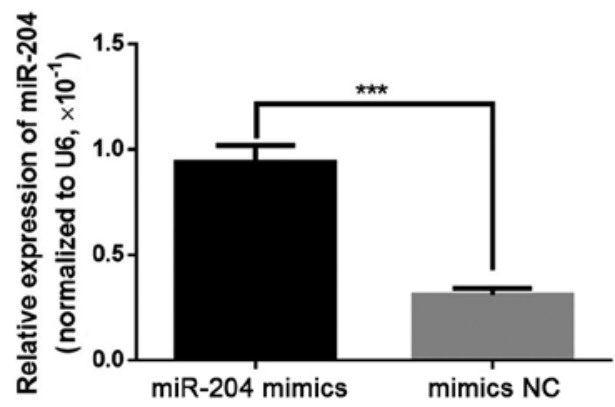

D

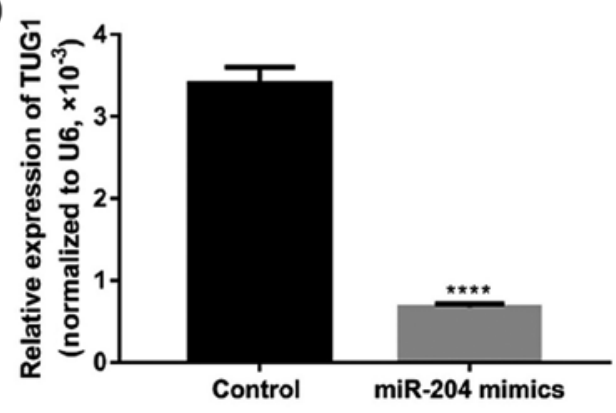

Figure 4. Long non-coding RNA TUG1 is a target of miR-204. (A) Diagram of binding sites between miR-204 and TUG1 was predicted by bioinformatics. (B) Dual luciferase assay was employed to illustrate the relative luciferase activity of miR-204 and the 3'-UTR of TUG1 in the 3T3-L1 cell lines. (C) The transfection efficacy of miR-204 in cells transfected with miR-204 mimics or mimics NC. (D) The expression of TUG1 following the overexpression miR-204 mimics in 3T3-L1 was detected by RT-qPCR, and U6 was used as the internal control. NC, negative control. ${ }^{* * *} \mathrm{P}<0.001$ and ${ }^{* * * *} \mathrm{P}<0.0001$, compared with the NC group.

in the diabetic mice when compared to those in the control group, and that the TUG1 injection markedly inhibited this augmentation, when compared to the NC group (Fig. 3B). Furthermore, IHC assay revealed that the expression of UCP-1 significantly decreased in the diabetic mice, and that the TUG1 injection significantly reversed these alterations in the diabetic mice (Fig. 3C). In addition, the underlying signaling pathway involved in this process was also investigated in these tissues. The results revealed that the expression of ATGL, PPAR $\alpha$, PGC- $1 \alpha$ and UCP-1 significantly decreased in the diabetic mice, and that the TUG1 injection significantly reversed these alterations in the diabetic mice $(\mathrm{P}<0.001$, Fig. 3D). The results of western blot analysis revealed that the expression of ATGL, PPAR $\alpha$, PGC-1 $\alpha$ and UCP-1, and the phosphorylation levels of AMPK and ACC were significantly downregulated in the diabetic mice, while the overexpression of TUG1 markedly attenuated the effects induced by diabetes (Fig. 3E).

TUG1 can interact with miR-204 and negatively regulate its expression. Considering the association between the expression of TUG1 and miR-204, the regulatory relation association ship between TUG1 and miR-204 was predicted, and an obvious miR-204 binding site was identified on the TUG1 mRNA (Fig. 4A). Dual luciferase reporter activity assay revealed that the overexpression of miR-204 by transfection with miR-204 mimic significantly reduced the reporter activity of TUG1, when compared to the NC group $(\mathrm{P}<0.05)$, while the overexpression of miR-204 had no marked effect on the reporter activity of MUT-TUG1, when compared to the NC group ( $\mathrm{P}>0.05$, Fig. 4B). In addition, transfection with miR-204 mimic significantly increased miR-204 expression, and the overexpression of TUG1 significantly inhibited the expression of miR-204 in the 3T3-L1 cells $(\mathrm{P}<0.05$, Fig. $4 \mathrm{C}$ and D). These findings suggest that TUG1 can directly and negatively affect the expression of miR-204.

TUG1 promotes fatty oxidation in adipocytes by negatively regulating miR-204. Following these investigations, the levels of fatty tissue oxidation and degradation-associated proteins were determined in the high glucose-induced 3T3-L1 fatty model. The results revealed that the expression levels of SIRT1, ATGL, PPAR $\alpha$, PGC- $1 \alpha$ and UCP-1, and the phosphorylation levels of AMPK and ACC, but not AMPK and ACC, significantly decreased in the model groups when compared to the control group. However, the overexpression of TUG1 significantly reversed the alteration in protein expression and phosphorylation in the 3T3-L1 model cells (Fig. 5). These findings suggest that TUG1 promotes fatty degradation and oxidation in adipocytes. However, the overexpression of miR-204 markedly attenuated the regulatory effects of TUG1 on fatty oxidation and degradation, namely, the decrease in the expression of SIRT1, ATGL, PPAR $\alpha$, PGC- $1 \alpha$ and UCP-1, and the phosphorylation levels of AMPK and ACC, without affecting the expression of AMPK and ACC in the 3T3-L1 cells (Fig. 5).

miR-204 directly inhibits the expression of SIRT1. According to bioinformatics prediction using TargetScan, it was predicted that SIRT1 and miR-204 shared some potential binding sites (Fig. 6A). Thus, dual luciferase reporter activity assay was used to confirm the regulatory association between SIRT1 and miR-204 in 3T3-L1 cells. As a result, the overexpression 
A
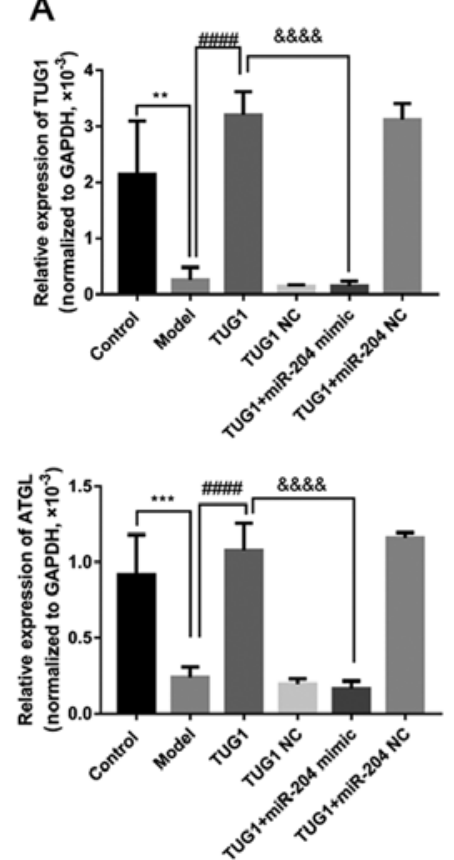
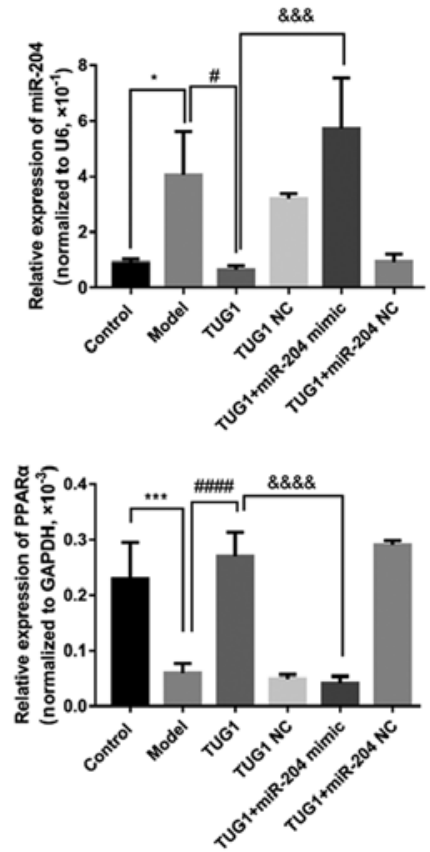

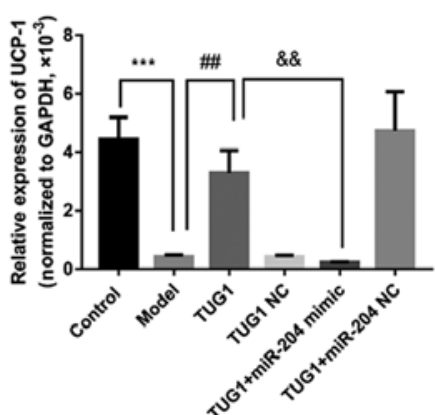

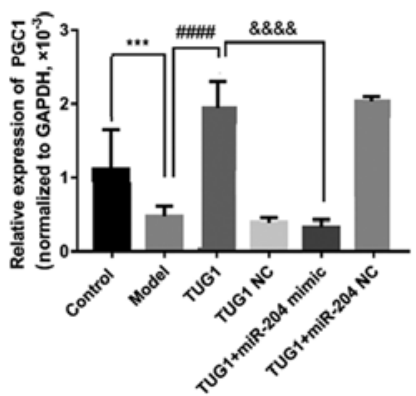
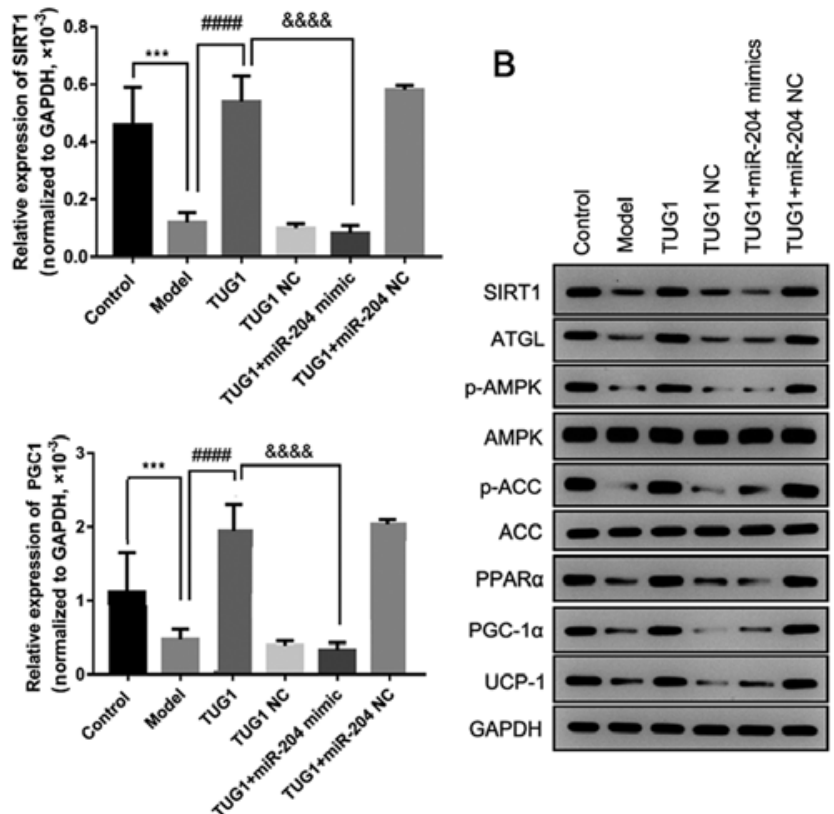

Figure 5. TUG1 promotes fatty oxidation in adipocytes by negatively regulating miR-204. (A) Relative expression of TUG1, miR-204, SIRT1, ATGL, PPAR $\alpha$, PGC- $\alpha$ and UCP1 was measured by RT-qPCR in 3T3-L1 cells after altering the expression of TUG1 and miR-204. (B) The protein expression of SIRT1, ATGL, p-AMPK, AMPK, p-ACC, ACC, PPAR $\alpha$, PGC-1 $\alpha$ and UCP-1 was evaluated by western blot analysis in 3T3-L1 cells after altering the expression of TUG1 and miR-204. ATGL, adipose triglyceride lipase; PGC-1 $\alpha$, peroxisome proliferator-activated receptor gamma coactivator 1- $\alpha$; PPAR $\alpha$, peroxisome proliferator-activated receptor $\alpha$; UCP-1, uncoupling protein-1; AMPK, AMP-activated protein kinase; ACC, acetyl-CoA carboxylase; NC, negative control ${ }^{*} \mathrm{P}<0.05,{ }^{* *} \mathrm{P}<0.01$ and ${ }^{* * *} \mathrm{P}<0.001$, compared with the control group; ${ }^{*} \mathrm{P}<0.05,{ }^{\# \#} \mathrm{P}<0.01$ and ${ }^{\# \# \# \#} \mathrm{P}<0.0001$, compared with the model group; \&\& $\mathrm{P}<0.01$, \&\&\& $\mathrm{P}<0.001$ and ${ }^{\& \& \&} \mathrm{P}<0.0001$, compared with the TUG1 group. Each assay, apart from western blot analysis, was repeated at least 3 times, and GAPDH or U6 was set as the internal control.

Hsa-miR-204: UCCGUAUCCUACUGUUUCCCUU
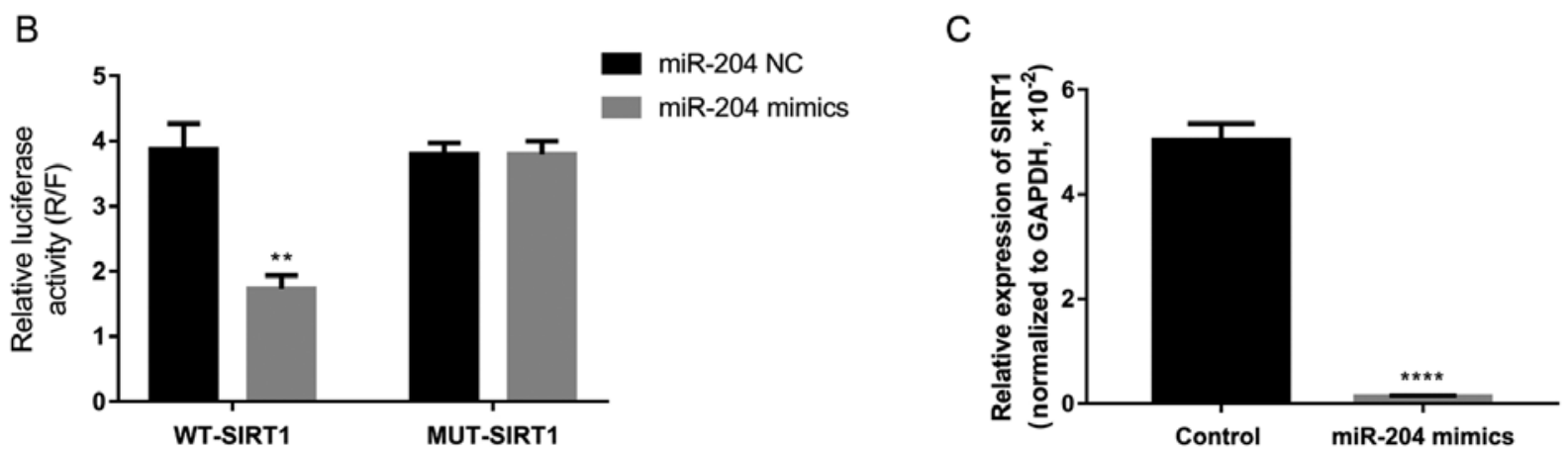

Figure 6. miR-204 directly downregulated the expression of SIRT1. (A) Prediction of binding sites between miR-204 and SIRT1 is presented in the diagram. (B) The confirmation of the regulatory association between miR-204 and the 3'-UTR of SIRT1 was proven by the dual luciferase activity in 3T3-L1 cells. (C) The expression of SIRT1 was detected by RT-qPCR following the overexpression of miR-204 mimics in 3T3-L1. NC, negative control. ${ }^{* *} \mathrm{P}<0.01$ and ${ }^{* * * * * *} \mathrm{P}<0.0001$, compared with the NC group. Each assay was performed at least in triplicate, and U6 was set as the internal control. 
A
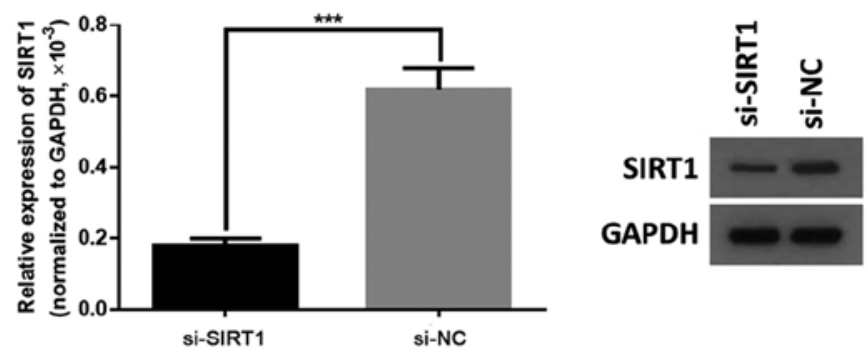

B
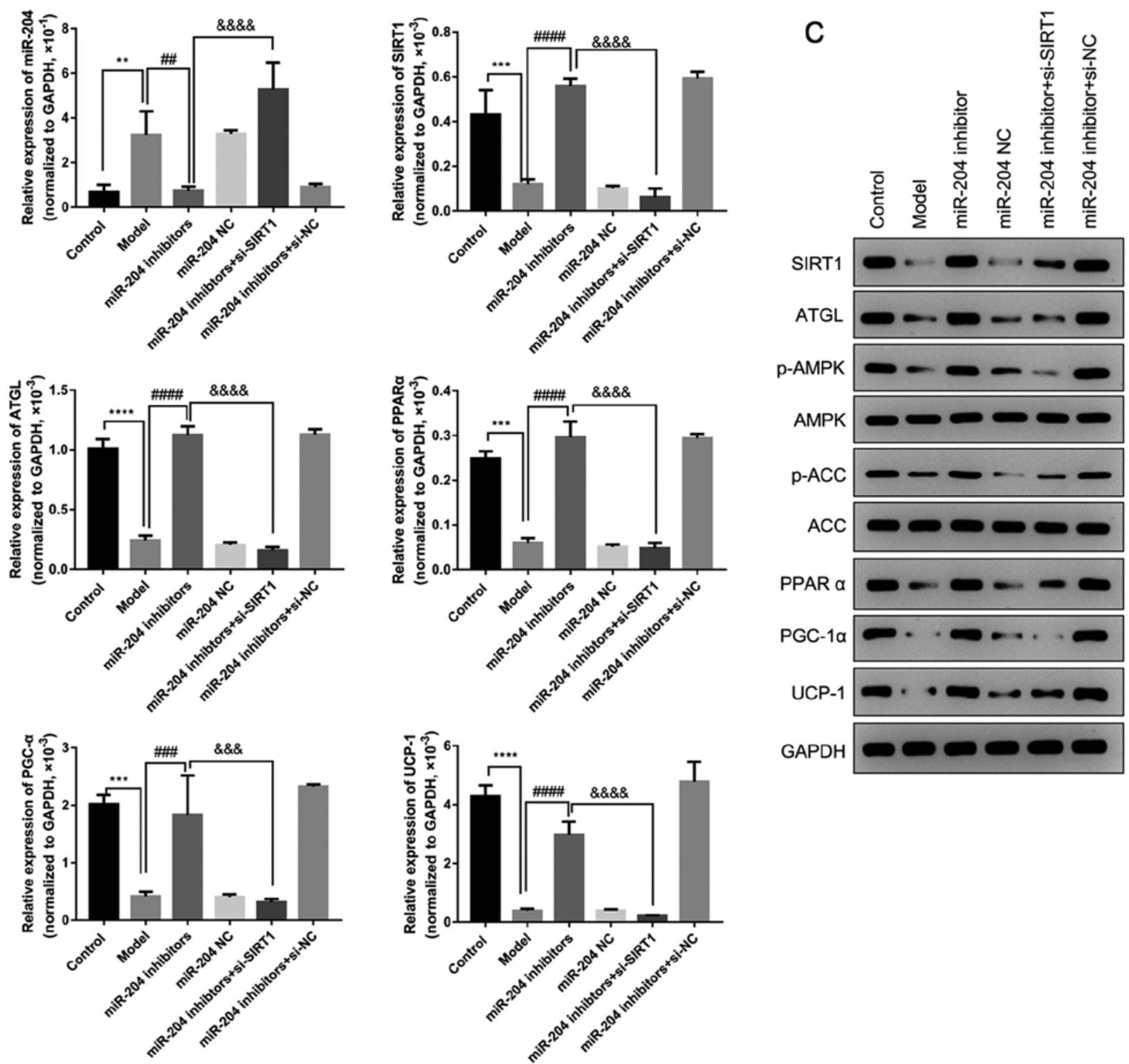

Figure 7. miR-204 inhibitor promotes fatty oxidation in adipocytes by upregulating SIRT1. (A) Expression of SIRT1 in model cells transfected with si-SIRT1 or si-NC. (B) Relative expression of miR-204, SIRT1, ATGL, PPAR $\alpha$, PGC- $\alpha$ and UCP-1 in 3T3-L1 cells was evaluated by RT-qPCR after altering the expression of SIRT1 and miR-204. (C) The protein expression of SIRT1, ATGL, p-AMPK, AMPK, p-ACC, ACC, PPAR $\alpha$, PGC-1 $\alpha$ and UCP-1 was evaluated by western blot analysis in 3T3-L1 cells after altering the expression of SIRT1 and miR-204. ATGL, adipose triglyceride lipase; PGC-1 $\alpha$, peroxisome proliferator-activated receptor gamma coactivator 1- $\alpha$; PPAR $\alpha$, peroxisome proliferator-activated receptor $\alpha$; UCP-1, uncoupling protein-1; AMPK, AMP-activated protein kinase; ACC, acetyl-CoA carboxylase; NC, negative control. ${ }^{* *} \mathrm{P}<0.01,{ }^{* * *} \mathrm{P}<0.001$ and ${ }^{* * * * *} \mathrm{P}<0.0001$, compared with the control group; ${ }^{\# \#} \mathrm{P}<0.01,{ }^{\# \# \#} \mathrm{P}<0.001$

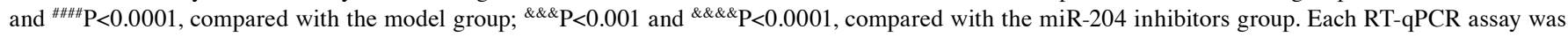
performed at least in triplicate, and GAPDH or U6 was set as the internal control.

of miR-204 significantly inhibited the reporter activity of WT-SIRT1, without affecting the activity of MUT-SIRT1 (Fig. 6B). In addition, the overexpression of miR-204 by transfection with miR-204 mimic significantly decreased the expression of SIRT1 in the 3T3-L1 cells (Fig. 6C). These findings indicate that SIRT1 is a direct target of miR-204. 
Inhibiting miR-204 promotes fatty oxidation in adipocytes by upregulating SIRT1. The present study then determined the effects of miR-204 on fatty oxidation. The results revealed that transfection with miR-204 inhibitor significantly elevated the expression of SIRT1, ATGL, PPAR $\alpha$, PGC-1 $\alpha$ and UCP-1, and the phosphorylation levels of AMPK and ACC in the 3T3-L1 cells, which were decreased by high glucose. However, the silencing of SIRT1 markedly attenuated the effects of miR-204 inhibitor, suppressing the expression of SIRT1, ATGL, PPAR $\alpha$, PGC- $1 \alpha$ and UCP-1, and the phosphorylation levels of AMPK and ACC in the 3T3-L1 cells (Fig. 7). These findings suggest that the inhibition of miR-204 promotes the high glucose-induced fatty oxidation in 3T3-L1 cells by upregulating SIRT1.

\section{Discussion}

Despite the development of management strategies, diabetes is becoming one of the most common health concerns worldwide, and the number of diagnosed cases is significantly increasing, particularly in developing countries (20). Considering this, multiple efforts have been made to explore the mechanism of diabetes, in order to providing new insight into the understanding of the pathogenesis of diabetes. In the present study, TUG1 and SIRT1 were significantly downregulated in diabetes, while the expression of miR-204 was significantly upregulated in diabetes. Therefore, the mechanisms of TUG1, miR-204 and SIRT1 were investigated in the present study, as well as the regulatory associations between these factors.

Recently, studies have documented that inhibiting the expression of TUG1 modulates the insulin secretion in mouse pancreatic $\beta$ cells $(21,22)$, suggesting that TUG1 may regulate the pathogenesis of diabetes. In the present study, TUG1 was identified to be significantly downregulated in diabetic mice, and the injection of the TUG1 lentivirus significantly attenuated the increase in body weight and adipose accumulation, and decreased the serum insulin, FGB, TG, glycerol and NEFA levels in diabetic mice. In addition, further investigations revealed that the overexpression of TUG1 markedly reversed the downregulation of ATGL, PGC-1 $\alpha$, PPAR $\alpha$ and UCP-1 expression, and AMPK and ACC phosphorylation in diabetic mice. As previously demonstrated, the silencing of TUG1 increases the apoptosis of pancreatic $\beta$ cells, but decreases serum insulin levels in vivo (21). Long et al reported that the overexpression of TUG1 rescued the expression of PGC-1 $\alpha$ and its transcriptional targets, in order to improve the mitochondrial biogenetics in diabetic nephropathy (23). Enhancing AMPK phosphorylation protects the diabetic heart against ischemia/reperfusion injury (24). Chronic and long-term exercise improves glucose and lipid metabolism by activating the AMPK-ACC signaling pathway (25). These findings suggest that IncRNA decreases adipose accumulation, in order to ameliorate the development of diabetes via the activation of the AMPK/ACC signaling pathway.

He et al reported that miR-204 promoted the audiogenic differentiation of human adipose-derived mesenchymal stem cells by regulating DVL3 in the Wnt/ $\beta$-catenin signaling pathway (26). In the present study, TUG1 was found to be a target of miR-204, and the overexpression of miR-204 significantly decreased the expression of TUG1. Furthermore, the overexpression of TUG1 suppressed miR-204, and increased
ATGL, PGC-1 $\alpha$, PPAR $\alpha$ and UCP-1 expression, and AMPK and $\mathrm{ACC}$ phosphorylation in diabetic mice. Yu et al reported that TUG1 could sponge miR-204 to promote the differentiation of osteoblasts by elevating the expression of Runx 2 in aortic valve calcification, which is a common complication in the obesity population (27). Taken together, these findings suggest that miR-204 promotes the development of diabetes by attenuating the expression of TUG1, and the activation of the AMPK/ACC signaling pathway.

The silencing of SIRT1 contributes to hepatic steatosis in obesity (28). In the present study, SIRT1 was found to be a of miR-204, and the overexpression of TUG1 significantly upregulated the expression of SIRT1. In addition, transfection with miR-204 inhibitor significantly increased the expression of SIRT1, ATGL, PGC-1 $\alpha$, PPAR $\alpha$ and UCP-1, and AMPK and ACC phosphorylation, whereas the silencing of SIRT1 could significantly reversed these effects. It has been reported that $\alpha$-lipoic acid modulates lipid metabolism by inducing SIRT1, and activating AMPK (29). In addition, $\alpha$-lipoic acid improves hepatic steatosis induced by a high-fat diet by modulating FoxO1 and Nrf2 in the SIRT1/LKB1/AMPK signaling pathway (30). These findings indicate that SIRT1 may inhibit the development of diabetes by reversing the effects of miR-204 through the SIRT1/AMPK/ACC signaling pathway.

In conclusion, the present study demonstrates that the abnormal upregulation of miR-204 promotes the development of diabetes, while the overexpression of TUG1 significantly promotes adipose oxidation and attenuates the procedure of diabetes via the upregulation of the SIRT1/AMPK/ACC signaling pathway. The present study also has some limitations. The time- and dose-dependent effects of miR-204 on the expression of SIRT1 and TUG1 were not investigated. Thus, the underlying molecular mechanisms of the role of miR-204 in diabetes and obesity warrant further in depth investigations.

\section{Acknowledgements}

Not applicable.

\section{Funding}

No funding was received.

\section{Availability of data and materials}

All data generated or analyzed during this study are included in this published article or are available from the corresponding author on reasonable request.

\section{Authors' contributions}

YZ, YM, MG and YP conceived and planned the experiments. YZ and YM carried out the experiments. YZ and MG performed the data analysis. YZ and YP wrote the manuscript. All authors read and approved the final manuscript.

\section{Ethics approval and consent to participate}

The animal protocols used in the present study were authorized by the Ethics Committee of the Shanghai General Hospital 
of Nanjing Medical University. The use of animals was also approved by the same Ethics Committee.

\section{Patient consent for publication}

Not applicable.

\section{Competing interests}

The authors declare that they have no competing interests.

\section{References}

1. Hoch E, Rusu V, Schreiber SL, Florez JC, Jacobs SB and Lander ES: Type 2 diabetes-associated variants disrupt function of SLC16A11, a proton-coupled monocarboxylate transporter, through two distinct mechanisms. FASEB J 31: S950.2, 2017.

2. Samuel VT and Shulman GI: Mechanisms for insulin resistance: Common threads and missing links. Cell 148: 852-871, 2012

3. Nunes MK, Silva AS, de Queiroga Evangelista IW, Filho JM, Gomes CN, Nascimento RA, Luna RC, de Carvalho Costa MJ, de Oliveira NF and Persuhn DC: Hypermethylation in the promoter of the MTHFR gene is associated with diabetic complications and biochemical indicators. Diabetol Metab Syndr 9: 84, 2017.

4. American Diabetes Association: 2. Classification and diagnosis of diabetes: Standards of medical care in diabetes-2018. Diabetes Care 41 (Suppl 1): S13-S27, 2018.

5. Rathwa N, Patel R, Palit SP, Ramachandran AV and Begum R Genetic variants of resistin and its plasma levels: Association with obesity and dyslipidemia related to type 2 diabetes susceptibility. Genomics 111: 980-985, 2018.

6. Pramanik S, Rathwa N, Patel R, Ramachandran AV and Begum R: Treatment avenues for type 2 diabetes and current perspectives on adipokines. Curr Diabetes Rev 14: 201-221, 2017.

7. Peirce V and Vidal-Puig A: Regulation of glucose homoeostasis by brown adipose tissue: Lancet Diabetes Endocrinol 1: 353-360, 2013.

8. Zhao S, Mugabo Y, Ballentine G, Attane C, Iglesias J, Poursharifi P, Zhang D, Nguyen TA, Erb H, Prentki R, et al: $\alpha / \beta$-Hydrolase domain 6 deletion induces adipose browning and prevents obesity and type 2 diabetes. Cell Rep 14: 2872-2888, 2016.

9. Perry RJ, Camporez JP, Kursawe R, Titchenell PM, Zhang D, Perry CJ, Jurczak MJ, Abudukadier A, Han MS, Zhang XM, et al: Hepatic acetyl CoA links adipose tissue inflammation to hepatic insulin resistance and type 2 diabetes. Cell 160: 745-758, 2015.

10. Timothy JP and Guy RA: Could lncRNAs contribute to $\beta$-cell identity and its loss in type 2 diabetes? Biochem Soc Trans 41: 797-801, 2013

11. Carter G, Miladinovic B, Patel AA, Deland L, Mastorides S and Patel NA: Circulating long noncoding RNA GAS5 levels are correlated to prevalence of type 2 diabetes mellitus. BBA Clin 4 102-107, 2015

12. Peng H, Zou L, Xie J, Wu H, Wu B, Zhu G, Lv Q, Zhang X, Liu S, Li G, et al: IncRNA NONRATT021972 siRNA decreases diabetic neuropathic pain mediated by the $\mathrm{P} 2 \mathrm{X} 3$ receptor in dorsal root ganglia. Mol Neurobiol 54: 511-523, 2017.

13. Leti $\mathrm{F}$ and DiStefano JK: Long noncoding RNAs as diagnostic and therapeutic targets in type 2 diabetes and related complications. Genes 8: 207, 2017.

14. Duan LJ, Ding M, Hou LJ, Cui YT, Li CJ and Yu DM: Long noncoding RNA TUG1 alleviates extracellular matrix accumulation via mediating microRNA-377 targeting of PPAR $\gamma$ in diabetic nephropathy. Biochem Biophys Res Commun 484: 598-604, 2017
15. Lei X, Zhang L, Li Z and Ren J: Astragaloside IV/ lncrna-TUg1/TraF5 signaling pathway participates in podocyte apoptosis of diabetic nephropathy rats. Drug Des Dev Ther 12: 2785-2793, 2018

16. Price NL, Gomes AP, Ling AJ, Duarte FV, Martin-Montalvo A, North BJ, Agarwal B, Ye L, Ramadori G, Teodoro JS, et al: SIRT1 Is required for AMPK activation and the beneficial effects of resveratrol on mitochondrial function. Cell Metab 15: 675-690, 2012.

17. Milne JC, Lambert PD, Schenk S, Carney DP, Smith JJ, Gagne DJ, Jin L, Boss O, Perni RB, Vu CB, et al: Small molecule activators of SIRT1 as therapeutics for the treatment of type 2 diabetes. Nature 450: 712-716, 2007.

18. Banks AS, Kon N, Knight C, Matsumoto M, Gutiérrez-Juárez R, Rossetti L, Gu W and Accili D: SirT1 gain of function increases energy efficiency and prevents diabetes in mice. Cell Metab 8: 333-341, 2008.

19. Yu SY, Dong B, Fang ZF, Hu XQ, Tang L and Zhou SH: Knockdown of lnc RNA AK 139328 alleviates myocardial ischaemia/reperfusion injury in diabetic mice via modulating miR-204-3p and inhibiting autophagy. J Cell Mol Med 22: 4886-4898, 2018

20. Gnavi R, Migliardi A, Maggini M and Costa G: Prevalence of and secular trends in diagnosed diabetes in Italy: 1980-2013. Nutr Metab Cardiovasc Dis 28: 219-225, 2017.

21. Yin DD, Zhang EB, You LH, Wang N, Wang LT, Jin FY, Zhu YN, Cao $1 \mathrm{H}$, Yuan QX, De W and Tang W: Downregulation of lncRNA TUG1 affects apoptosis and insulin secretion in mouse pancreatic $\beta$ cells. Cell Physiol Biochem 35: 1892-1904, 2015.

22. Cao LH, Yin DD, Xia CC, Wang N and De W: Function of lncRNA TUG1 in insulin secretion from pancreatic beta cells. Xian Dai Sheng Wu Yi Xue Jin Zhan 25: 4847-4851, 2017 (In Chinese).

23. Long J, Badal SS, Ye Z, Wang Y, Ayanga BA, Galvan DL, Green NH, Chang BH, Overbeek PA and Danesh FR: Long noncoding RNA Tug1 regulates mitochondrial bioenergetics in diabetic nephropathy. J Clin Invest 126: 4205-4218, 2016.

24. Paiva MA, Rutter-Locher Z, Gonçalves LM, Providência LA, Davidson SM, Yellon DM and Mocanu MM: Enhancing AMPK activation during ischemia protects the diabetic heart against reperfusion injury. Am J Physiol Heart Circ Physiol 300: H2123-H2134, 2011

25. Yi X, Cao S, Chang B, Zhao D, Gao H, Wan Y, Shi J, Wei W and Guan Y: Effects of acute exercise and chronic exercise on the liver leptin-AMPK-ACC signaling pathway in rats with type 2 diabetes. J Diabetes Res 2013: 946432, 2013.

26. He H, Chen K, Wang F, Zhao L, Wan X, Wang L and Mo Z: MiR-204-5p promotes the adipogenic differentiation of human adipose-derived mesenchymal stem cells by modulating DVL3 expression and suppressing wnt/ $\beta$-catenin signaling. Int $\mathbf{J}$ Mol Med 35: 1587-1595, 2015.

27. Yu C, Li L, Xie F, Guo S, Liu F, Dong N and Wang Y: LncRNA TUG1 sponges miR-204-5p to promote osteoblast differentiation through upregulating Runx2 in aortic valve calcification. Cardiovas Res 114: 168-179, 2017.

28. Zhanguo G, Zhang J, Kheterpal I, Kennedy N, Davis RJ and Ye J: Sirtuin 1 (SIRT1) protein degradation in response to persistent c-jun n-terminal kinase 1 (JNK1) activation contributes to hepatic steatosis in obesity. J Biol Chem 286: 22227-22234, 2011.

29. Chen WL, Kang CH, Wang SG and Lee HM: $\alpha$-Lipoic acid regulates lipid metabolism through induction of sirtuin 1 (SIRT1) and activation of AMP-activated protein kinase. Diabetologia 55: 1824-1835, 2012

30. Yang Y, Li W, Liu Y, Sun Y, Li Y, Yao Q, Li J, Zhang Q, Gao Y, Gao L and Zhao J: Alpha-Lipoic acid improves high-fat diet-induced hepatic steatosis by modulating the transcription factors SREBP-1, FoxO1 and Nrf2 via the SIRT1/LKB1/AMPK pathway. J Nutr Biochem 25: 1207-1217, 2014. 